\title{
Desnutrición infantil, coeficiente de desarrollo y su relación con el medio ambiente: un estudio piloto
}

\author{
Infant undernourishment, the development coefficient and its relation to the \\ environment: a pilot study
}

\author{
M. de la Luz Alvarez*, Ximena Concha**, Margarita Elordi**, Cecilia Lamilla**, \\ Carolina Ramos**, Paulina Perez*
}

\begin{abstract}
ALVAREZ, M. L. et al.. Desnutrición infantil, coeficiente de desarrollo y su relación con el medio ambiente: un estudio piloto. Rev. Saúde públ., S. Paulo, 25: 282-8, 1991. Fueron estudiados los factores socioculturales que podrían estar influyendo en el desarrollo psicomotor normal del lactante desnutrido. La muestra consistió en 32 díadas madrelactante: 16 tenían CD normal (Grupo A) y 16 $C D$ bajo lo normal (Grupo B) según el Test de Bailey aplicado a los lactantes recién ingresados a un Centro de Recuperación Nutricional. Los resultados muestran que había algunas diferencias en los antecedentes de los lactantes: Los lactantes del Grupo A eran producto de un embarazo deseado (p<.05), y fueron menos separados del lado de su madre ( $p<.007)$ que aquellos del Grupo B. No se encontraron diferencias en los antecedentes socioculturales y demográficos de las madres. El medio externo de los lactantes fue diferente en los aspectos observados en el barrio: hubo menos aspectos negativos en el Grupo A que en los del B ( $p<.001)$. El medio interno de los lactantes, también fue diferente en lo relacionado con la madre. Madres del Grupo A percibían más afección por parte de su pareja ( $p<.008$ ), eran más sensitivas (sentimientos de alegría y de sufrimientos) ( $p<.003$ ) y estimulaban más a su hijo $(\mathrm{p}<.004)$ que aquellas del Grupo B. Estos resultados muestran que el medio externo e interno en el cuál se desenvolvían los lactantes con $C D$ normal y $C D$ bajo lo normal eran diferentes. Esto podría dar cuenta de las diferencias en el desarrollo psicomotor entre lactantes desnutridos pertenecientes a un bajo estrato socioeconómico.
\end{abstract}

Descriptores: Desarrollo infantil. Factors socioeconómicos. Trastomos de la nutrición infantil.

\section{Introduccion}

Numerosos estudios han demostrado que la desnutrición infantil produce un retardo en el crecimiento y éste puede ser más o menos severo dependiendo del grado de nutrición $n^{3,4,6,10,14,16,17,25}$.

También se ha dicho que la desnutrición afectaría el desarrollo psicomotor del lactante, y por lo tanto, dejaría secuelas en el desarrollo psicológico posterior ${ }^{7,18,21,26}$. Sin embargo, esto último no es tan evidente cuando se evalúa el Coeficiente de Desarrollo (CD) de los lactantes desnutridos.

En un estudio realizado sobre el lenguaje gestual de la madre durante el acto de darle de comer a su hijo lactante con desnutrición severa $(60-70 \%$ de nutrición según Tablas del NCHS) ${ }^{15}$, se encontró que había un $35 \%$ de lactantes con $C D$ nor$\mathrm{mal}^{2,24}$. Todos estos niños estaban viviendo en sus

\footnotetext{
* Instituto de Nutrición de la Universidad de Chile.

** Escuela de Trabajo Social del Instituto Professional de Santiago.
}

Separatas/Reprints:

M. de la Luz Alvarez - Casilla 138 - 11, Santiago , Chile. hogares. Actualmente, se encuentra al momento del ingreso de los niños internados en los Centros de Recuperación Nutricional de la Corporación para la Nutrición Infantil (CONIN) un CD normal, aproximadamente en un $30 \%$.

Esta situación nos motivó para investigar que factores de tipo sociocultural podrían estar incidiendo en el desarrollo psicomotor normal de un lactante desnutrido. En este momento, prácticamente no se encuentran lactantes con desnutrición primaria severa en nuestro país, y CONIN está recuperando a los lactantes con desnutrición moderada (70$80 \%$ de nutrición).

\section{Material y Metodo}

La muestra está formada por 32 díadas madrelactante: 16 con CD normal (Grupo A) y 16 con CD bajo lo normal (Grupo B) según el Test de Bailey aplicado al ingreso del lactante al Centro de Recuperación Nutricional. El cien por ciento del grupo A fue calificado por dicho test como normal y el $31,2 \%$ como dudoso y $68,8 \%$ como anormal en el grupo B. Todos los lactantes provenían del 
área urbana de Santiago (Chile). Ambos grupos pertenecían a un nivel socioeconómico (NSE) bajo, las madres no diferían en nivel de escolaridad, ni en edad ( $\overline{\mathrm{X}} 19,62$ grupo A y 20,12 grupo $B)$, ni en años de convivencia con su pareja ( $\bar{X}$ 3,06 grupo A y 4.37 grupo B). Los grupos fueron pareados por edad del lactante. El $\bar{X}$ de edad del grupo A fue de 11.688 meses y la del grupo B fue de 12.688. La DS en el primer grupo es de 5.029 y en el segundo de 4.785 meses, pero no hay diferencia entre ambos grupos $(\mathrm{p}<0,5579)$.

Las técnicas aplicadas fueron:

1) Entrevistas: a) Encuesta Socioeconómica para medir el NSE con un instrumento que discrimina en poblaciones relativamente homogéneas ${ }^{1}$. Esta escala incluye 13 variables cuyo puntaje es de 1 a 6 puntos. Las variables que incluye dicho instrumento son: número de personas que viven en el hogar, presencia del padre del lactante en el hogar, escolaridad y actividad del jefe de hogar, propriedad de la casa en que viven, calidad de la casa, sistema de abastecimiento de agua, disposición de excretas, seguridad social, promiscuidad, pieza independiente para cocinar, recolección de basuras y algunos bienes en el hogar los cuáles dan un solo puntaje. b) Encuesta Sociocultural para medir algunos antecedentes del embarazo, parto y separaciones de la madre antes de que el lactante ingrese a CONIN; aspectos afectivos en su relación de pareja y expresión de sentimientos (alegrías y penas).

2) Observaciones estructuradas: a) Medición del medio ambiente interno y externo que rodea al lactante: a') en el hogar: presencia de colores predominantes en el jardín si había, por ej. plantas o flores; en los muros, (externos de la casa e internos), o sea el color que tenían, lo mismo en los muebles; si había orden, ej. que no hubiera cosas tiradas que no correspondieran a lo que un niño puede hacer uso tales como juguetes, y la presencia de animales domésticos. a") en el barrio: presencia a 6 cuadras a la redonda de aspectos negativos (basurales, canales abiertos, venta de alcohol clandestina, y otros) y positivos (centros de abastecimientos, canchas de deportes, agrupaciones comunitarias, templos, movilización). Todo esto se marcaba con una $X$ significando la presencia de lo que se buscaba. b) Relación madre-lactante: frecuencia y calidad de la estimulación que la madre daba a su hijo internado en el Centro de Recuperación Nutricional. Se observó durante $10 \mathrm{~min}$. lo que la madre hacía del punto de vista verbal (hablarle, cantarle), gestual (caricias, besos) y lúdico (juegos ya fuera con juguetes o con sus propias manos) al momento de visitar por segunda vez al niño. Se hizo así, para dar tiempo a la madre a que se habituara a la nueva situación.

Para realizar esta observación se actuó en pareja y sin que la madre observada se diera cuenta que ella era objeto de estudio, pues se actuaba dentro de la sala como una persona más del Centro de Recuperación Nutricional. Las conductas observadas se registraban en una hoja diseñada para este fin con las variables antes mencionadas en cada indicador específico. Una vez terminada la observación se comparaba lo registrado en cada hoja y sólo se aceptó aquellas observaciones en que había un $90 \%$ de coincidencia en la conducta observada. Las observadoras fueron entrenadas previamente en la misma situación de los Centros de Recuperación de CONIN.

\section{Procedimiento}

Lo primero que se hizo fue la observación de la relación madre-lactante sin saber a que grupo pertenecía el niño a fin de evitar algún sesgo en la re-colección de la información. Luego se visitó a la madre en su domicilio para realizar las entrevistas y la observación de la casa y del barrio. Posteriormente se conoció el resultado del Test de Bailey para saber en que grupo se colocaría al lactante. El Test de Bailey fue aplicado por especialistas de CONIN en los Centros de Recuperación Nutricio-nal dentro de la semana de ingresso del lactante. Esto es parte de la rutina de atención que se da al lactante y no hubo interferencia con la observación.

El análisis de datos se hizo a través de îndices:

- NSE está compuesto de 65 puntos máximo y un mínimo de 13. Se considera NSE bajo cuando se obtiene un puntaje de 40 y más puntos. En esta población que es considerada deprivada, los puntajes fluctuaron entre 45 y 60 puntos correspondiendo a un nivel medio bajo y muy bajo.

- Expresión de penas (encuesta sociocultural) está compuesto por 6 indicadores cuyo puntaje fluctuó entre un máximo de 6 puntos y un mínimo de 0 . La escala es de: $0-2=$ alta expresión de penas; $2-4=$ moderada expresión de penas; $4-6=$ baja ex presión de penas. Esto se obtuvo a través de una pregunta que decía: podría nombrar 3 penas o cosas que la hayan hecho sufrir el año pasado? Se consignaba el hecho que fuera capaz de mencionar una o más penas y el tipo de ella (material, afectiva o ambas). Si la madre era capaz de mencionar 3 penas y las tres de tipo afectivo obtenía 0 puntos, lo cuál indicaba que era capaz de expresar externamente sus penas. En la medida que no era capaz de decirlo, su puntaje aumentaba y demostraba menor capacidad de expresión.

- Expresión de alegrías está constituído por 6 indicadores cuyo puntaje va de un máximo de 6 
puntos a un mínimo de 0 . La escala es de: $0-2=$ baja expresión de alegrías; $2-4$ = moderada expresión de alegrías; 4-6 = alta expresión de alegrías. Esto se obtuvo preguntando a la madre que dijiera 3 alegrías o acontecimientos que la hicieron feliz el año anterior. También se registró el tipo de la alegría como en el caso anterior.

- Índice sentirse rechazada: este índice comprende una serie de preguntas sobre la vida pasada desde el nacimiento hasta que formó su propia familia. Se preguntó si se había sentido rechazada, por quien (padre, madre, abuelos, tíos hermanos) y cómo se lo demostraron (formas: materiales, afectivas o ambas). Los puntajes flucturon entre 5 y 0 . A mayor puntaje menor es el rechazo percibido por la madre en su infancia y adolescencia.

- Aspectos negativos del barrio: está formado por 7 indicadores como los mencionados anteriormente. El puntaje oscila entre 0 y 7 puntos. Cero significa la ausencia de lo observado. La escala va de: $0-2.3$ = barrio no negativo; $2.3-4.6$ = barrio más o menos negativo; $4.6-7=$ barrio negativo.

- Aspectos positivos del barrio: está formado por 8 indicadores según los ítems señalados anteriormente. El puntaje va de 0 a 8 puntos indicando 0 punto la ausencia de lo observado. La escala va de: $0-2.6$ = barrio no positivo; $2.6-5.2=$ barrio más o menos positivo; 5.2-8 = barrio positivo.

- Estimulación materna: está formado por todas las conductas verbales (hablar, cantar, repetir sonidos al niño), gestuales (besar, sonreir, acariciar) y lúdicas (pasar objetos para que juegue el niño, pasearlo en la pieza, jugar con él) que realizó la ma- dre en el tiempo de la observación. El máximo de conductas observadas fueron 105 y el minimo 14. Se consideró baja estimulación aquella que obtuvo entre 1 y 50 puntos y alta, aquella que presentó entre 51 y 105 puntos.

Se aplicaron las pruebas de Fisher por ser una muestra pequeña, el $\mathrm{t}$ de Student y la Regresión Logística Multiple.

\section{Resultados}

Primeramente se presentaráalgunos antecedentes del lactante. Más de la mitad de los lactantes del grupo A provienen significativamente más de un embarazo deseado contra menos de la mitad de los del grupo $B(p<0,05)$. Más de la mitad de las madres de los lactantes del grupo A recibieron alimentación suplementaria durante el embarazo contra menos del tercio de aquellas del grupo $B(p<0,05)$. Las semanas de gestación no difieren entre ambos grupos, lo mismo el tipo de parto que fue mayoritariamente normal $(87,6 \%$ vs $81,2 \%)$. Tampoco difieren los grupo en el peso de nacimiento que fue sobre 2.500 grs. en ambos grupos. Se encontró que los lactantes del grupo A tuvieron significativamente menos separaciones del lado de su madre que los lactantes del grupo B ( $p<$ .007), antes de ingresar al Centro de Recuperación Nutricional de CONIN. Estas separaciones de 15 días tienden a ser menos en el grupo A que en el B ( $p<$ 0,09 ). Finalmente, el promedio peso/ edad del lactante al ingreso a CONIN fue más alto en el grupo $\mathrm{A}$ que en el $B(p<0,05)$, presentando ambos una desnu-

Tabla 1. Antecedentes generales del lactante, área urbana, Santiago, Chile.

\begin{tabular}{|c|c|c|c|}
\hline \multirow[t]{2}{*}{ Antecedentes } & \multicolumn{2}{|c|}{ Grupo } & \multirow[t]{2}{*}{ Test } \\
\hline & A & $\mathbf{B}$ & \\
\hline Embarazo Deseado & $68,8 \%$ & $43,8 \%$ & $F p<0.05$ \\
\hline $\begin{array}{l}\text { Suplementacion Alimentaria } \\
\text { Durante el Embarazo }\end{array}$ & $62,5 \%$ & $31,2 \%$ & $F p<0.05$ \\
\hline $\bar{X}$ Semanas de Gestación & $36,3 \%$ & $35,8 \%$ & $t \mathrm{~ns}$ \\
\hline Parto Normal & $87,6 \%$ & $81,2 \%$ & ns \\
\hline Peso $\bar{X}$ de Nacimiento & 2.612 grs. & 2.575 grs. & t ns \\
\hline Separación Madre-Lactante & $37,5 \%$ & $68,8 \%$ & $F p<0.007$ \\
\hline Separación de 15 dias y más & $50,0 \%$ & $90,9 \%$ & $F p<0.09$ \\
\hline $\begin{array}{l}\bar{X} \text { Peso/Edad al Ingreso al } \\
\text { Centro de Recuperación }\end{array}$ & 7.688 grs. & 7.050 grs. & $t<0.05$ \\
\hline $\begin{array}{l}\bar{X} \text { Peso/Edad al Ingreso al } \\
\text { Centro de Recuperación }\end{array}$ & $82.75 \mathrm{cms}$. & $82.62 \mathrm{cms}$ & $t$ ns \\
\hline
\end{tabular}


trición moderada. Sin embargo, no hay diferencia en el promedio peso/talla entre ambos grupos (Tabla 1).

La Tabla 2 presenta el medio ambiente externo del lactante. En relación a los colores, la única diferencia encontrada es en el azul y amarillo. El primero se encuentra significativamente menos en el grupo A y el segundo, está significativamente más presente en dicho grupo en relación con el grupo B $(p<.01)$. Los aspectos negativos del barrio encontrados fueron la presencia significativamente menor de basurales y de canales abiertos en el grupo A en relación al grupo B ( $p<0,0009$ y $p$ $<0,02$ respectivamente). El índice de aspectos negativos del barrio fue significativamente inferior en el grupo A en relación al grupo B ( $p<0,003)$. Los aspectos positivos del barrio tales como la presencia de centros de abastecimientos (p $<0,001$ ), canchas de deportes ( $p<0,01$ ), agrupaciones $(p<0,01)$ y templos, iglesias $(p<0,02)$ fueron significativamente mayores en el grupo $A$ en relación al grupo B. A nivel del índice de aspectos positivos las familias del grupo A presentaron significativamente mayores aspectos positivos que aquellas del grupo B ( $p<0,001)$.

La Tabla 3 señala la situación de la madre y su relación con el lactante. Un tercio de las madres del grupo A percibia afecto por parte de su pareja contra ninguna en el grupo $B(p<0,008)$. La mayoria de las madres del grupo A fueron capaces de manifestar las alegrías vividas el año anterior contra menos de la mitad de aquellas del grupo $B(81,3 \%$ vs $43,7 \%)$. Las alegrías manifestadas fueron más afectivas en el grupo A que aquellas del grupo B $(68,7 \%$ vs $37,5 \%)$. Las penas también fueron más expresadas por las madres del grupo A que aquellas del grupo B $(75 \%$ vs $37,5 \%)$ y fueron de tipo afectivas $(\mathrm{p}<0,03)$. Finalmente las madres del grupo A estimulan significativamente más a su hijo lactante que aquellas del grupo B ( $<<0,004)$.

La Tabla 4 presenta la Regresión Logística Múltiple realizada a fin de explicar, en parte, el $C D$ del lactante desnutrido. El índice de expresión de penas está correlacionado negativamente con el CD: a mayor índice de expresión de penas (o incapacidad de expresión) sentidas por la madre, menor es el CD del lactante $(p<0,01)$. La percepción de la madre de haber sido castigada en su familia se correlaciona positivamente con el $C D$ $(p<0,02)$. El índice de aspectos negativos del barrio se correlaciona negativamente con el $C D$, mientras más negativo es el barrio (o sea, mayor puntaje obtenido) más bajo es el CD del lactante $(\mathrm{p}<0,03)$. Finalmente, el índice de rechazo percibido por la madre en su familia de origen presenta una tendencia a correlacionarse positivamente con el $C D(p<0,07)$. Es decir, a mayor puntaje
Tabla 2. Ambiente externo que rodea al lactante, área urbana, Santiago, Chile.

\begin{tabular}{|c|c|c|c|}
\hline \multirow[t]{2}{*}{ Ambiente } & \multicolumn{2}{|c|}{ Grupo } & \multirow{2}{*}{$\begin{array}{c}\text { Test } \\
\mathrm{F} \\
\mathrm{P}\end{array}$} \\
\hline & $\begin{array}{c}A \\
(\%)\end{array}$ & $\begin{array}{c}B \\
(\%)\end{array}$ & \\
\hline $\begin{array}{l}\text { colores: } \\
\text { Amarillo } \\
\text { Azul }\end{array}$ & $\begin{array}{l}37,5 \\
87,5\end{array}$ & $\begin{array}{l}68,7 \\
68,7\end{array}$ & $\begin{array}{l}<0.01 \\
<0.01\end{array}$ \\
\hline $\begin{array}{l}\text { Aspectos Negativos del Barrio: } \\
\text { Basurales } \\
\text { Canales Abiertos } \\
\text { Indice Menores Aspectos }\end{array}$ & $\begin{array}{r}6,2 \\
12,5\end{array}$ & $\begin{array}{l}62,5 \\
50,0\end{array}$ & $\left\{\begin{array}{l}<0.0009 \\
<0.02\end{array}\right.$ \\
\hline Negativos & 81,3 & 43,7 & $7<0.003$ \\
\hline $\begin{array}{l}\text { Aspectos Positivos del Barrio: } \\
\text { Centros de Abastecimientos } \\
\text { Canchas de Deportes } \\
\text { Agrupaciones Comunitarias } \\
\text { Templos e Iglesias } \\
\text { Indice de Mayores Aspectos } \\
\text { Positivos }\end{array}$ & $\begin{array}{r}100,0 \\
93,8 \\
87,5 \\
87,5 \\
\\
100,0\end{array}$ & $\begin{array}{l}50,0 \\
56,2 \\
43,8 \\
50,0 \\
50,0\end{array}$ & $\begin{array}{l}<0.001 \\
<0.01 \\
<0.01 \\
<0.02 \\
<0.001\end{array}$ \\
\hline
\end{tabular}

Tabla 3. Situación de la madre y su relación con el lactante, área urbana, Santiago, Chile

\begin{tabular}{|c|c|c|c|}
\hline \multirow{2}{*}{$\begin{array}{l}\text { Situación de } \\
\text { la Madre }\end{array}$} & \multicolumn{2}{|c|}{ Grupo } & \multirow{2}{*}{$\begin{array}{c}\text { Test } \\
F \\
P\end{array}$} \\
\hline & $\begin{array}{c}A \\
(\%)\end{array}$ & $\begin{array}{c}B \\
(\%)\end{array}$ & \\
\hline $\begin{array}{l}\text { Mayor Percepción de Afecto } \\
\text { Por Parte de su Pareja }\end{array}$ & 33,3 & 0,0 & $<0.008$ \\
\hline Expresan más Alegrias & 81,3 & 43,7 & $<0.003$ \\
\hline $\begin{array}{l}\text { Las Alegrías Son } \\
\text { de Tipo Afectivas }\end{array}$ & 68,7 & 37,5 & $<0.007$ \\
\hline Expresan más Penas & 75,0 & 37,5 & $<0.003$ \\
\hline $\begin{array}{l}\text { Las Penas Son más de Tipo } \\
\text { Afectivas }\end{array}$ & 56,3 & 18,7 & $<0.03$ \\
\hline $\begin{array}{l}\text { Alta Estimulación (Verbal y } \\
\text { Lúdica) }\end{array}$ & 37,5 & 6,3 & $<0.004$ \\
\hline
\end{tabular}

Tabla 4. Regresión logística múltiple en el $C D$ de lactantes desnutridos, area urbana, santiago, chile

\begin{tabular}{lcc}
\hline Variables & B & $p$ \\
\hline Media & 1,88 & $<0,19$ \\
Indice Expresión de Penas & $-5,64$ & $<0,01$ \\
Haberse Sentido Castigada & 3,47 & $<0,02$ \\
$\begin{array}{l}\text { Indice Aspectos Negativos del } \\
\text { Barrio }\end{array}$ & $-3,41$ & $<0,03$ \\
$\begin{array}{l}\text { Indice de Haberse Sentido } \\
\text { Rechazada }\end{array}$ & 3,01 & $<0,07$ \\
\hline
\end{tabular}


del Índice de Rechazo significa que la madre fue menos rechazada y a su vez tiende el niño a tener mayor $C D$.

\section{Discusion y Conclusiones}

La aceptación del retardo en el desarrollo psico-motor del lactante desnutrido ha impedido llevar a cabo otro tipo de investigaciones que demuestren que esta afirmación no es tan cierta. En estudios realizados hace años atrás se encontró que los lactantes con desnutrición severa presentaban un $C D$ normal, pero esto quedó a un nivel restringido de difusión, ya que iba contra todo lo conocido y aceptado hasta ese momento ${ }^{13}$. Con el tiempo, la CONIN a través de sus Centros de Recuperación Nutricional también encontró que alrededor del $30 \%$ de los lactantes desnutridos presentaban un CD normal y no sabia a qué atribuirlo. Por esto, se estudió fundamentalmente el medio en el cual se desenvolvía el lactante. El fin fue detectar que factores podrían asociarse y correlacionarse con el desarrollo psico-motor del lactante, ya que este desarrollo se basa principalmente en los estímulos que recibe ${ }^{19,20}$.

Primeramente se encontró que los antecedentes de los lactantes difieren en diversos aspectos. Los lactantes con CD normal presentan aspectos positivos tan importantes como: haber sido un hijo deseado ( $p<0,05)$, haber sufrido muchísimo menos separaciones de la madre antes de ingresar a un Centro de Recuperación Nutricional de CONIN ( $p$ $<0,007)$ y que estas separaciones tienden a ser. más cortas en tiempo $(p<0,09)$. Muchas de estas separaciones se debieron a hospitalizaciones del lactante, lo cual indica la mayor morbilidad y fueron anteriores a la internación en el Centro de Recuperación Nutricional de CONIN. Otras se debieron a que la madre dejaba al lactante en casa de la abuela para que se lo cuidara. Pese a estas separaciones, los lactantes del grupo A se vieron menos afectados por ella como es habitual en muchos casos de hospitalizaciones prolongadas ${ }^{8}$, porque habría otros factores que lo favorecieron.

También se encontró que durante el embarazo de los lactantes con $C D$ normal, la madre recibió más suplementación alimentaria que las otras $(\mathrm{p}<$ 0.05 ). Esto podría indicar que estuvo más controlada por el equipo de salud que las otras madres y que podría haber recibido más información acerca de los cuidados que debería dar a su hijo, tanto durante el embarazo, como del recién nacido. Esto es parte de la rutina de trabajo de las matronas y de las enfermeras de los Consultorios Periféricos.

El hecho que no se haya encontrado ninguna diferencia respecto a las semanas de gestación, ni al tipo de parto, ni en el peso de nacimiento, sig- nificaría que las diferencias en el CD posiblemente no podrían ser atribuídas en este caso, a estos factores. Sin embargo, la relación peso/edad, al momento de ingresar al Centro de Recuperación Nutricional de CONIN, es significativa, aunque dentro del rango de desnutrición moderada. Los lactantes del grupo A presentaron un peso superior al de aquellos del grupo $B(p<0,05)$, pero no se encontró diferencia en la relación peso/talla. El hecho de no encontrar diferencia significativa entre ambos grupos en relación al NSE, señalaría que éste "per se" no condicionaría el medio ambiente en el cual se desenvuelve el lactante y que existiría otros factores que estarían influyendo. El NSE medido por los 13 indicadores que se usaron en este caso está mostrando las características inmediatas del jefe de hogar y de la vivienda, lo cuál no invalida las características externas del medio, en este caso, del barrio en que está ubicada la familia. El medio externo que rodea al lactante es significativamente diferente entre los dos grupos. Por ejemplo, a nivel de colores se encontró que el azul está más presente en el grupo A $(\mathrm{p}<0,01)$ y el amarillo menos $(\mathrm{p}<0,01)$. Es indudable que los colores ejercen una estimulación visual y en este caso, el color más estimulante se encontró en los lactantes con CD normal.

Luego, los aspectos menos negativos y más positivos del barrio ( $p<0,003$ y $p<0,001$ respectivamente) se encontraron en el grupo A. Esto podría indicar que las condiciones del barrio ayudarían a la madre a sacar al niño fuera del hogar por diferentes razones: llevarlo cuando ella hace las compras, pues tiene centros de abastecimientos cercanos; llevarlo a la cancha de foot-ball los fines de semana, ya que esta es una entretención muy difundida en los barrios y es gratuita; llevarlo a la iglesia o templo, pues es habitual asistir a los servicios si se practica una religión. Esto se corrobora con la correlación negativa encontrada: a mayores aspectos negativos del barrio, menor $C D$ presenta el lactante $(\mathrm{p}<0,03)$. Estos resultados están acordes con otros estudios los cuáles valoran la calidad del barrio ${ }^{13}$.

Las características de tipo sociodemográficas de la madre, tampoco difieren. Los grupos presentaron similar baja escolaridad, edad y años de convivencia con su pareja. Esto señalaría que las diferencias de $C D$ del lactante se deberían a otras características propias de la madre tales como: mayor equilibrio afectivo en las madres del grupo A que en aquellas del grupo B. Las primeras sienten mayor afección por parte de su pareja ( $p<$ 0,0008 ) lo cual podria significar que al sentirse queridas, ellas también transmitan ese cariño a su hijo; son capaces de expresar penas y alegrías ( $p<$ 0,003 ) de tipo afectivo lo cual indicaría una mayor sensibilidad que las otras madres. 
Esto se reafirma con la correlación encontrada respecto a la expresión de penas $(p<0,01)$.

Pero también es importante destacar la relación observada entre la madre y el lactante. Esta indicó que existía una alta estimulación por parte de la madre hacia el lactante en aquellas madres del grupo A $(p<0,004)$. Se ha demostrado que el lactante necessita ser estimulado para lograr su desarrollo psicomotor $5,9,11,12,22,23$, por lo tanto, estas madres al actuar en forma más estimulante, junto con presentar un ambiente externo más positivo, tener más capacidad de expresar sus penas y alegrías y tener una tendencia a haber sido menos rechazadas durante su infancia y adolescencia, podrian haber contribuído en el $\mathrm{CD}$ normal que presentó el lactante del grupo $\mathrm{A}$.

ALVAREZ, M. L. et al. [Desnutrição infantil, coeficiente do desenvolvimento e sua relação com o meio ambiente: um estudo piloto.] Rev. Saúde publ., S. Paulo, 25: 282-8, 1991. Estudam-se os fatores sócio-culturais que poderiam estar influenciando no desenvolvimento psicomotor normal do lactente desnutrido. A amostra consistiu de 32 pares mãe-criança: 16 com coeficiente de desenvolvimento $(C D)$ normal (grupo $A$ ) e $16 \mathrm{com}$ CD abaixo do normal (grupo B) de acordo com o teste de Bailey aplicado a lactentes que ingressavam a um centro de recuperação nutricional. Havia diferenças nos antecedentes das crianças: os lactentes do grupo A eram fruto de gravidez desejada $(p<0,05)$, e foram menos separados de suas mães $(p<0,007)$ que os do grupo $B$. Não se encontravam diferenças nos antecedentes demográficos e sócio-culturais das mães. Em relação ao meio ambiente externo observado no bairro de residência, havia menos aspectos negativos no grupo $A$ que no $B(p<$ $0,001)$. O meio interno também foi diferente em relação às mães: As do grupo $\mathrm{A}$ recebiam mais carinho por parte do companheiro $(p<0,008)$, eram mais sensíveis (sentimentos de alegria e sofrimento $)(p<0,003)$ e estimulavam mais os seus filhos $(p<0,006)$ que as do grupo $B$. Os resultados mostraram que os meios internos e externos no qual se deservolvem crianças com $C D$ abaixo do normal eram diferentes. Isto poderia explicar as diferenças no desenvolvimento psicomotor em desnutridos pertencentes a baixos níveis sócio-econômicos.

Descritores: Desenvolvimento infantil. Fatores sócioeconômicos. Distúrbios da nutrição infantil, complicações.

ALVAREZ, M. L. et al [Infant undernourishment, the development coefficient and its relationship to the environment: a pilot study.] Rev. Saúde públ., S. Paulo, 25: $282-8,1991$. The socio-cultural factors that might be influencing the normal psychomotor development of the undernourished infant are studied. The sample consisted of 32 mother-infant dyads: 16 having normal DQ (Group A) and 16 having a below normal DQ (Group B) according to Bailey's Test applied to infants who entered a Nutritional Recuperation Center. Results showed that there were some differences in the infants' backgrounds: infants of Group A were the product of a wished-for pregnancy $(p<.05)$, and were separated less from their mother's side $(\mathrm{p}<.007)$ than was the case in Group B. No differences were found in the socio-cultural and demographic background of the mothers. The infants' external environment was different in aspects observed within the neighborhood: there were fewer negative aspects in Group A than in Group B $(p<.003)$ and more positive in Group A than in B $(p<.001)$. The infant's internal environment as related to the mother was also different. Mothers of Group A perceived more affection from their partner $(p<.008)$, were more sensitive (to feelings of joy and suffering) $(p<.003)$ and stimulated their infant $(p<.004)$ more than those of Group B. These results show that the internal and external environments in which the infant with normal and below normal DQ evolves were different between the two groups. This could explain the differences in psychomotor development among undernourished infants belonging to the lower socioeconomic strata.

Keywords: Child development. Socioeconomic factors. Infant nutrition disorders.

\section{References}

1. ALVAREZ, M.L.; WURGAFT, F.; SALAZAR, M.E. Mediciones de nivel socioeconomico bajo urbano en familias con lactante desnutrido. Arch. latinoamer. Nutr., 32: $650-62,1982$.

2. ALVAREZ, M.L.; WURGAFT, F.; WILDER, H. Non verbal language in mothers with malnourished infants: a pilot study. Soc. Sci, - Med., 16: 1365-9, 1982.

3. ASTBURY, J.; ORGILL, A.A.; BAJUK, B.; YU, V.Y.H. Sequelae of growth failure in appropiate for gestational age, very low birth-weigh infants. Develop. Med. Child Neurol. 28: 472-9, 1986.

4. BRAZELTON, B.T.; TRONICK, E.; LECHTIG, A.; LASKY, R.E.; KLEIN, R. E. The behavior of nutritionally deprived Guatemalan infants. Develop. Med. Child Neurol., 19: 364-72, 1977.

5. BROFENBRENNER, U. Ecology of the family as a context for human development research perspectives. $\mathrm{De}$ velop. Psycol., 22: 723-42, 1986.

6. CATER, J.I. Correlates of low birth weight. Child Care Hith Develop., 6: 267-77, 1980.

7. CELEDON, J.M. \& ANDRACA, I. de. Psychomotor development during treatment of severely marasmic infants. Early hum. Develop., 3: 276-285, 1979.

8. CELEDON, J.M.; CSASZAR, D.; MIDDLETON, J.: ANDRACA, L. de. The effect of treatment of marasmic in. fants according to age of admission. J. ment. Defic. Res., 24: 27-35, 1980.

9. COHN, J.F. \& TRONICK, E.Z. Mother-infant face-to-face interaction: the sequence of dyadic status at 3,6 and 9 months. Develop. Psycol., 23: 68-77, 1987.

10. CRAVIOTO, J. \& DELICARDIE, E. La malnutrición precoz en la primera infancia \& algunas de suas posteriores repercusiones en los individuos y en la comunidad. Aliment. y Nutr. 2: 2-12, 1976.

11. ESTRADA, P.; ARSENIO, W.F.; HESS, R.D.; HOLLOWAY, S.D. Affective quality of the mother-child relationship: longitudinal consequences for children's school-relevant cognitive functioning. Develop. Psychol., 23: 210-5, 1987. 
12. FEIRING, C.; FOX, N.A.; JASKIN, J.; LEWIS, M. The relation between social support, infant risk status and mother-infant interaction. Develop. Psychol., 23: 400-5, 1987.

13.GARBARINO, J. \& SHERMAN, D. High-risk neigbordhoods and high risk families: the human ecology of child maltreatment. Child Develop., 51: 188-98, 1980.

14. GRAVES, P.L. Nutrition and infant behavior: a replication study in the Katmandu, Valley Nepal. Amer. J. clin. Nutr. 31: 541-51, 1978.

15. HAMILL, P.V.V.; DRIZD, T.A.; JOHNSON, C.L.; REED, R.B.; ROCHE, A.F.; MOORE, W.M. Physical growth: National Center for Health Statistics percentiles. Amer. J. clin. Nutr., 32: 607-29, 1979.

16. JOHNSTON, F.E.; NEWMAN, B.; CRAVIOTO, J.; DELICARDIE, E.; SCHOLL, T. A factor analysis of correlates of nutritional status in Mexican children, birth to 3 years. In: Greene L.S. \& Johnston, F.E., eds. Social and biological predictors of nutritional status physical growth and neurological development. New York, Academic Press, 1980. p. 291-307.

17. LASKY. R.E.; KLEDN, R.E.; YARBROUGH, C.; ENGLE, P.L.; LECHTIG, A.; MARTORELL, R. The relationship between physical growth and infant behavioral development in rural Guatemala. Child. Develop., 52: 219-26, 1981.

18. LASKY, R.E.; KLEIN, R.; YARBROUGH, C.; SELLARS, M.J.; KAGAN, J. Social interactions of Guatemalan infants. J. Cross cult. Psychol., 14 (1): 17-28, 1983.

19. OSOFSKY, J.D. \& CONNORS, K. Mother-infant interaction: an integrative view of a complex system. In: Osofsky, J., ed. The handbook of infant development. New
York, John Wiley, 1979. p. 519-48.

20. PARKE, R.D. Children's home environments: social and cognitive effects. In: Alıman, I. \& Wohlwill, J.F., eds. Children and environment: human behavior and environment. New York, Plenum, 1978. v. 3. p. 33-81.

21. POLIJT, E. \& LEWIS, N. Nutrition and educational achievement. Part II: Correlations between nutritional and behavioral test indicators within populations where malnutrition is not a major public healih problem. Food Nutr. Bull. 2 (4): 33-7, 1980.

22. RUDDY, M.G. \& BORNSTEIN, M.H. Cognitive correlates of infant attention and matemal stimulation over the first year of life. Child Develop., 53: 183-8, 1982.

23. SANDEEP, P. \& PUSHPA, M. Deprivation and cognitive development in children. Child Psychiatry Quart. 14 (2): 47-54, 1981 .

24. WURGAFT, F. Factores socioculturales y su relación con la desnutrición del lactante: estudio en madres de nivel socioeconomico bajo. Santiago, 1978. [Tesis Licenciado en Antropologia-Universidad de Chile].

25. ZESKIND, P. S. \& RAMEY, C.T. Fetal malnutrition: an experimental study of its consequences on infant development in two caregiving environments. Child Develop. 49: $1155-62,1978$

26. ZESKIND, P.S. \& RAMEY, G.T. Preventing intellectual and interactional sequelae of fetal malnutrition: a longitudinal, transactional and synergistic approach to development. Child Develop., 52: 213-8, 1981.

Recebido para publicação em 12/11/1990. Reapresentado em 29/05/1991. Aprovado para publicaçäo em 3/06/1991. 